\title{
A Descriptive Study on Types of Violence, It's Effects on Ante Natal Patient's Health and Pregnancy Outcomes
}

\section{Naila Nasr Malik ${ }^{1 *}$, Shamim Akhtar ${ }^{1}$, Humaira Bibi ${ }^{2}$, Rabia Kareem ${ }^{3}$, Bushra Khan $^{4}$ and Farhat R Malik ${ }^{5}$}

${ }^{1}$ Associate Professor, Ob/gyn unit, Kuwait Teaching Hospital, Peshawar Medical College, Riphah International University, Pakistan

${ }^{2}$ Assistant Professor, Ob/gyn unit, Kuwait Teaching Hospital, Peshawar Medical College, Riphah International University, Pakistan

${ }^{3}$ Senior Registrar, Ob/gyn unit, Mercy Teaching Hospital, Peshawar Medical College, Riphah International University, Pakistan

${ }^{4}$ Specialist A, Omran Hospital, UAE

${ }^{5}$ Professor Community Health Sciences, Peshawar Medical College, Riphah

International University, Pakistan

*Corresponding Author: Naila Nasr Malik, Associate Professor, Ob/gyn unit, Kuwait Teaching Hospital, Peshawar Medical College, Riphah International University, Pakistan.
Received: September 21, 2021

Published: October 21, 2021

(C) All rights are reserved by Naila Nasr

Malik, et al.

\begin{abstract}
Objectives: To determine the common types of violence, their effects on pregnant female's health and pregnancy outcomes.

Methodology: A prospective cross-sectional study was conducted in six-month duration by selecting 130 antenatal patients of all trimesters coming from OPD, labor room as an emergency as well as elective patients. The patient's clinical record was analyzed for obstetrical complications and through systematic sampling technique, all the patients were scrutinized for types of violence through a validated questionnaire. All the recorded information entered in SPSS Version-21 with descriptive and inferential analysis with the computation of frequencies, percentages, chi-square tests with the cut-off limit for the significance set at 0.05.

Results: One hundred and thirty pregnant women participated in this study with mean average age of $25.12+7.89$ years. The verbally abused females were $(n=54,41.5 \%)$, followed by physical abused $(n=20,15.4 \%)$ and $(n=44,33.8 \%)$ without disclosing the reason behind violence respectively. Hardly a single female was abused on daily basis ( $\mathrm{n}=1,0.8 \%$ ), whereas majority faced the violence on daily basis $(n=41,31.5 \%)$ followed by monthly frequency $(n=25,19.2 \%)$ respectively. The common problems disclosed were family problems $(n=24,18.5 \%)$ and financial issues $(n=18,13.8 \%)$. Most of the females didn't disclose the effects of violence ( $n=56,43.1 \%)$, with emotional distressed females $(n=39,30 \%)$ and lack of care of pregnancy $(n=14,10.8 \%)$ respectively.

Conclusion: Intimate partner violence is a frequent public health problem indicating immediate measures to curb this situation. The results showed a majority of verbally abused, emotionally disturbed women and family problems as the root cause behind.

Keywords: Pregnancy; Physical Abuse; Domestic Violence; Emotional Abuse; Pregnant Women; Risk Factors; Birth Outcomes; Intimate Partner Violence; Prevalence
\end{abstract}

Citation: Naila Nasr Malik., et al. "A Descriptive Study on Types of Violence, It's Effects on Ante Natal Patient's Health and Pregnancy Outcomes". Acta Scientific Medical Sciences 5.11 (2021): 111-116. 


\section{Introduction}

Violence against females is a significant social and public health issue as well as a human rights violation. As per WHO statistics, $30 \%$ of females are subjected to this tragedy worldwide, with one quarter suffering from intimate partners among the 15-49-year age group with adverse physical, psychological and sexual harm [1]. Intimate partner violence or domestic violence is defined as an abnormal abusive threatening behavior by the nearest relation with lifelong trauma that leads to serious and long-term maternal and fetal adverse birth outcomes. Millions of women of reproductive age in developing as well as the developed world are victims with negative consequences on their mental health [2]. The most common type of violence women experienced at any stage is by their sexual intimate partner during pregnancy, threatens not the pregnant females in the form of abortion, miscarriage, pre-eclampsia, gestational diabetes, and placental abruption but to the unborn fetus as well associated with low birth, premature births, intrauterine growth retardation, with leading causes of neonatal morbidity and mortality $[3,4]$. IPV types include slapping, kicking, pushing, beating, and forcing sexual intercourse as well as psychological abusing like insults, humiliation, threats, controlling behaviors, monitoring movements, and restricting access to health care services [4].

Intimate partner violence has been researched in Pakistan since 1990, but no definitive data was available. A systematic review done in 2014, took 23 research articles of quantitative, qualitative, and mixed-method designs to probe into the problem, but all the studies were done in hospital settings. All results of these studies reported the prevalence of IPV, men as the perpetrators and women as victims. The common causes and factors behind this were families without children, financial instability, husband's beating nature, and infertility among couples. The common type of violence reported was a sexual, physical, and psychological type. Findings from the studies revealed a low prevalence of IPV in KPK (Khyber Pakhtunkhwa,Province of Pakistan) due to low literacy and less empowerment of females. Unfortunately, no study was conducted on the true meaning of IPV from people's perspectives [5]. An umbrella review conducted in this regard took 58 studies, but the final selection included 12 systematic and 5 meta-analyses only. Most of them were cross-over studies and only single assessments of women during the antenatal period. This review gave an overview of the global prevalence of IPV, with most physical and sexual type violence among women. Although these were recent reviews but of low quality with AMSTAR2 recommendations and without capturing the total global burden of the disease under discussion. These showed only one out of two and one of 50 females suffer physical and psychological violence [6].

A Ph.D. dissertation was conducted upon 519 females due for their abortions at a hospital, with a purpose to assess the prevalence of the common types of violence experienced by the females along with the association of substance use and depression. Results of this survey showed a $12.3 \%$ prevalence of IPV among the couples where men were unemployed and women were in employment status. A positive association was found with substance abuse indicators like alcohol, drug use, and other stuff, higher depressive illnesses, and less social support. This study did not take into account the type and source of social support [7]. Quality of life among physically and psychologically abused was an important public health issue for females and their off-springs. As these have drastic implications upon pregnancy outcomes, so the women needed special extra care during their antenatal period. This study assessed subscales of health in terms of physical functioning, vitality, social functioning, emotional stability on a scale of $0-100$. Therefore, ante-natal women need urgent screening, prevention, and detection of abuse to save them from drastic consequences [8].

To the best of the researcher's knowledge so far and internet browsing, no reliable data was available regarding Pakistani statistics on this topic. Therefore, this study was aimed to have a baseline data in relation to the exposure of intimate partner violence among ante-natal women in this area with the determination of common types of violence's, their causes, effects on the patient's health, pregnancy outcomes, and association with the variables of interest, to highlight the subject matter and provide a sound platform for services and therapeutic programs for this purpose.

\section{Methodology}

A prospective cross-sectional study was conducted in six-month duration time after being ethically approved by the institution on $7^{\text {th }}$ March- 2018 with (IRB Approval Number: Prime/IRB/2017-180090).

The sample size was calculated as 129 by using $14 \%$ prevalence of domestic violence in our population, with $95 \%$ confidence in- 
terval and 6\% margin of error according to the (WHO software for sample size determination). Non- probability consecutive sampling technique was employed for recruiting the patients in the study.

All married antenatal women of all trimesters coming from obstetrical OPD, labor room as an emergency, as well as elective case and willing to answer the required validated research questionnaire, were included in the study with all the antenatal women who declined to be interviewed were excluded from the study. Informed consent was taken from the participants, meeting the inclusion criteria. For ethical reasons, including potential retaliation from disclosure, women were interviewed in private.

\section{Operational definition}

Intimate partner violence [2]:

"It is defined as an abnormal behavior by a near and dear relative with adverse long term trauma of maternal and fetal birth outcomes".

The research tool was adapted from the widely used validated Abuse Assessment Screen (AAS) II, with locally adapted questions to assess natal outcomes and history of abuse in previous pregnancy as well. Sociodemographic indicators included husband's and wife's education with the profession as well as female patient's period of gestation, parity, and gravida.

The patient's clinical record was analyzed for obstetrical complications and all the patients were scrutinized for types of violence, frequency of violence, cause of violence, and effect of violence on their health through a validated questionnaire (AAS-II).

All the recorded information entered in SPSS Version- 21 with descriptive and inferential analysis with the computation of frequencies, percentages, and generation of graphs. Association of husband's education was tested against types and causes of violence through the application of chi-square tests with cut-off limit for the significance set at 0.05 .

\section{Results}

A total of 130 pregnant women participated in this study with the age range of 18 to 40 years. They were relatively young, with a mean average age of $25.12+7.89$ years. Most of the patients belonged to Peshawar ( $n=99,76 \%)$, and Afghanistan $(n=15,12 \%)$. The uneducated participants were $(n=40,31 \%)$, followed by
Middle education ( $\mathrm{n}=26,20 \%$ ), and hardly few participants were highly educated to the university level $(n=15,11.5 \%)$. Whereas husband's education level was relatively high, more than half of the patient's husbands ware educated ( $n=60,53.7 \%$ ) while few of husbands were educated $n=60$. Uneducated $(n=21,16.2 \%)$ and hardly few were highly educated to the level university ( $n=15$, $11.5 \%$ ) respectively.

Majority of the females were unemployed $(n=82,63.1 \%)$ with few employed ( $n=4,3.2 \%)$, however few of the females didn't mention their occupational status ( $\mathrm{n}=44,33.8 \%)$. The unemployed husbands were $(n=7,5.4 \%)$, few of the them didn't mention their jobs $(n=24,18.5 \%)$ while remaining were employed ( $n$ $=69,76.1 \%)$.

The female's majority were pregnant more than 1 time, $(n=74$, $80 \%)$, few were pregnant only for $1^{\text {st }}$ time $(n=21,16.2 \%)$, while only $\mathrm{n}=5(3.8 \%)$ didn't reported any number of pregnancies respectively. Most of the females were multipara $(n=64,49.2 \%)$ and few didn't report any $(n=37,28.5 \%)$ respectively.

Most of the females were verbally abused ( $n=54,41.5 \%)$, followed by physical abuse $(\mathrm{n}=20,15.4 \%)$ with some females who didn't disclose any violence to anyone $(n=44,33.8 \%)$ respectively. Hardly a single female was abused on daily basis $(n=1,0.8 \%)$, whereas majority were occasionally facing the violence $(n=41$, $31.5 \%)$ followed by monthly frequency $(n=25,19.2 \%)$ respectively. Details are shown in figure 1 and 2.

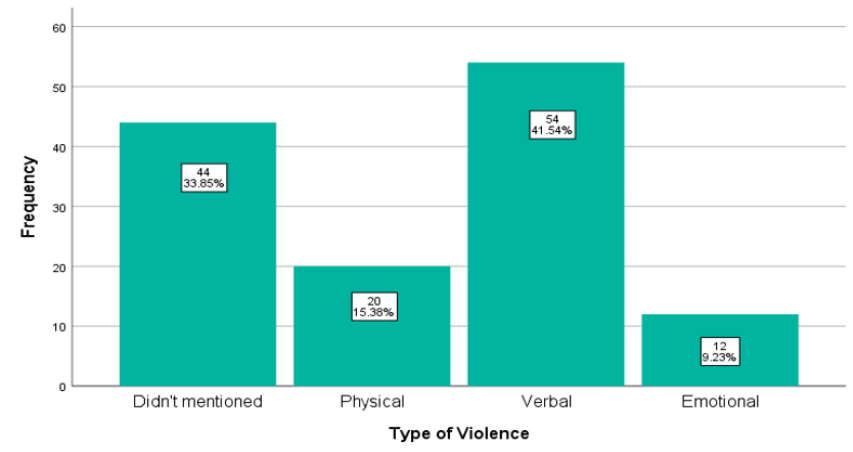

Figure 1: Common Types of Domestic Violence in Pregnancy (n=130). 


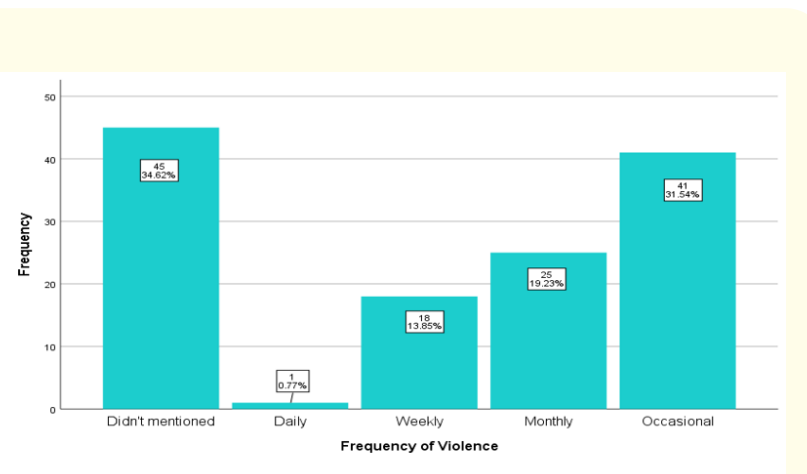

Figure 2: Frequency of Domestic Violence in Pregnancy

$$
(n=130) \text {. }
$$

Most of the females didn't disclose the cause of violence to anyone $(n=43,33.1 \%)$, while majority of the females reported other causes, like financial, family problems, on child gender, forced and lack of satisfaction with marriage ( $n=37,28.5 \%$ ), followed by mainly family problems $(n=24,18.5 \%)$, then financial issues $(n=$ $18,13.8 \%$ ) respectively. Most of the females didn't disclose the effects of violence $(n=56,43.1 \%)$. However, emotional distress was found in $(n=39,30 \%)$ and lack of care of pregnancy among $(n=14$, $10.8 \%)$. Further details are given in table 1.

\begin{tabular}{|l|c|c|}
\hline S. No & Causes & N (\%) \\
\hline 1 & Didn't Mentioned & $43(33.1 \%)$ \\
\hline 2 & Financial Problems & $18(13.8 \%)$ \\
\hline 3 & Family Problems & $24(18.5 \%)$ \\
\hline 4 & Female Gender on Ultra Sound & $2(1.5 \%)$ \\
\hline 5 & Lack of Satisfaction from Marital & $2(1.5 \%)$ \\
\hline 6 & Life & $4(3.1 \%)$ \\
\hline 7 & Forced Marriages & $37(28.5 \%)$ \\
\hline
\end{tabular}

Table 1: Causes of Domestic Violence in Pregnancy $(n=130)$.

The Chi-square test was applied to determine the association among types and causes of violence in pregnancy and husband's education. The results on both violence and causes of violence in pregnancy with husband's education showed a non-significant association $(p>0.05)$ respectively.

\section{Discussion}

The present study results of intimate partner violence depicted high magnitude of verbal abused women (41.4\%), least (15.3\%) of physical and emotional violence (9.3\%) victims. However, maximum women never revealed the type of violence that was unfortunate reality (33.4\%). The frequency of domestic violence came out to be occasional (31\%) however majority (34.6\%) never mentioned it. Frequency of women daily abused turned out to be $0.77 \%, 13.85 \%$ on weekly and $19.23 \%$ on monthly basis. The most common cause of domestic violence was family problems i.e. $18.50 \%$, followed by $13.1 \%$ financial issues and $33.1 \%$ never revealed anything. These results when compared with the international and national figures showed underestimation of the public problem in women. Also men think it as their birth right to degrade women, beat them owing it to their cultural norms and rights.

A hospital based case-control study interviewed 954 mothers with history of intimate partner violence and a mean age of the participants as $28.37 \%+6.25$ Years. The results of this study showed $68.6 \%$ cases, $26.9 \%$ controls as victims of global tragedy with $54.7 \%$ cases and $8 \%$ controls reported with physical type of violence. Sexual violence was found in $22 \%$ cases and $11 \%$ controls. Multivariate Logistic regression showed three times more adverse birth outcomes in women exposed to intimate partner violence with LBW and preterm deliveries and five times more vulnerable in case of physical type of violence. No significant associations were found with type of the violence and aggressive behaviors of the husbands. All these results were not in consistency with the present study as designs were completely different with analysis pattern as well as the mean age of the participants [3].

A descriptive study with a mean age of the females as $29.09+$ 4.99 Years, showed enormously excellent knowledge regarding the topic among the participants. The females experienced $30.4 \%$ IPV during their life, with physical (62.07\%), sexual (57.30\%), economical (48.50\%) and psychosocial (53.50\%) violence. A peculiar finding was that $61 \%$ of the women did not do anything regarding IPV due to fear. These findings were not contrary to the present study results as physical violence was top listed whereas verbal abuse was at top in the present study. Moreover, the present study never checked respondents level of knowledge of IPV [4]. A published systematic review and meta- analysis gave a low quality prevalence of IPV as 1.6- 78\% physical abuse and psychological 
type as 1.8-67.4\%. These results were contradictory to the present results as verbal abuse was among the highest reported type of the violence [6].

An Iranian study assessed quality of life in abused pregnant women. The results showed $68 \%$ women with physical and psychosocial IPV during their ante- natal period, significantly affecting the quality of life and physical functioning of their systems [8]. However, the present study only assessed causes, types of IPV with effects of birth outcomes and did not take into account the quality of life of the victims.

Although pregnancy is a physiological state but it may not be a joyful time for every pregnant woman globally. A Malawi study on ante- natal women showed 59\% women subjected to domestic violence with a mean age of 25.5 years among 15 - 45 years. The frequency of violence was $14 \%$ physical, 28\% sexual and 29\% emotional. A significant association ( $P>0.05$ ) was found among the women abused and a child abused in the same house with a strong association of domestic violence and pregnant women. However, no significant relationship found between occupation, income and other variables of interest. All the results of this study were in correlation with the present study except the type of violence and significant associations shown. However, results of the mean age and relationship of violence with education and occupation were the same [9]. Similarly, another study in Tabriz- Iran also took ante natal females and assessed quality of life with prevalence of IPV. Results showed one third women with IPV and emotional abuse was the top listed one as $32.8 \%$ followed by sexual $(12.4 \%)$ and physical violence as (4.8\%) [10]. However, the present study showed verbal abuse as the most common type but never assessed quality of life.

An IPV study showed $26.7 \%$ women being slapped, kicked, hit and beaten by husband with strong independent association with IPV in pregnancy and daily alcoholic habit of husband [11]. This study had uneven distribution of the risks. These results were not in consistency as type of violence had different shape as compared to the existing study and no associations were found with daily habits of husbands in the present study. A mixed-method study identified prevalence and types of violence with its associations in pregnancy as well as postpartum period through Abuse Assessment Screening (AAS), Hurt, Insulted, threatened with Harm and Screamed (HITS),
Partner Violence Screen (PVS) and Women Abusing Screening Tool (WAST). Focus group discussions were conducted for this purpose [12]. This study is different from the present study as they used three different type of questionnaire with FGD however, present study only used AAS with no FGDs and post- partum group was missing in this study.

An Observational Study on literate Omani women revealed one fifth pregnant women as victims of IPV (1-2\%), with significant association of physical IPV with un-intended pregnancy, higher rates of depression, insomnia and husbands as perpetuators behind this violence. There was no impact of IPV on birth outcomes [13]. These findings are consistent with the present study however, this study reported low physical IPV and with reported verbal abuse and severe birth outcomes. A qualitative meta- analysis strongly recommended health practitioners readiness towards domestic violence, with available facilities to cater such patients [14]. This study results were contrary to the present study as it was a meta- analysis and the present one was a descriptive study only.

\section{Limitations of this study were as follows}

- This study was a cross-sectional one with a small sample size, so the results cannot be extrapolated.

- It did not include post- partum group of females.

- Only one research tool used in the present study, that was not enough to cover all aspects of domestic violence.

\section{Conclusion}

The findings of this study revealed a highest number of verbally abused pregnant women with family problems as the common cause behind it.

\section{Acknowledgement}

Special thanks to all the participating antenatal women and the staff who supported us throughout research. We are really obliged by all the cooperation rendered in this regard.

\section{Bibliography}

1. World Health Organization. "Violence against women". Key Facts (2021).

2. ACOG; Clinical- Intimate Partner Violence (2021). 
3. Berhanie E., et al. "Intimate partner violence during pregnancy and adverse birth outcomes: a case-control study". Reproductive Health 16.1 (2019): 22.

4. Oche OM., et al. "Intimate Partner Violence in Pregnancy: Knowledge and Experiences of Pregnant Women and Controlling Behavior of Male Partners in Sokoto, Northwest Nigeria". International Journal of Reproductive Medicine 2020 (2020): 7626741.

5. Ali PA., et al. "Intimate Partner Violence in Pakistan: A Systematic Review". Trauma, Violence and Abuse (2014): 1-17.

6. Román- Gálvez RM., et al. "Prevalence of Intimate Partner Violence in Pregnancy: An Umbrella Review". International Journal of Environmental Research and Public Health 18.707 (2021): 1-13.

7. Baydoun HA. "Intimate partner violence, employment and social support among women seeking elective abortion services In Iowa [PhD Thesis- Dissertation]". Graduate College of University of Iowa (2009).

8. Tavoli Z., et al. "Quality of life in women who were exposed to domestic violence during pregnancy". BMC Pregnancy and Childbirth 16 (2016): 1-28.

9. Chasweka R., et al. "Isn't pregnancy supposed to be a joyful time? A cross-sectional study on the types of domestic violence women experience during pregnancy in Malawi". Malawi Medical Journal 30 (2018): 191-196.

10. Naghizadeh S., et al. "Domestic violence and its relationship with quality of life in pregnant women during the outbreak of COVID-19 disease". BMC Pregnancy and Childbirth 21.1 (2021): 88.

11. Clarke S., et al. "Intimate partner violence in pregnancy: a cross-sectional study from post-conflict northern Uganda". BMJ Open 9.11 (2019): e027541.

12. Sánchez OdR., et al. "Violence against women during pregnancy and postpartum period: A Mixed Methods Study Protocol". BMJ Open 10 (2020): e037522; 1-7.
13. Al Shidhani NA., et al. "Prevalence, Risk Factors and Effects of Domestic Violence Before and During Pregnancy on Birth Outcomes: An Observational Study of Literate Omani Women". International Journal of Women's Health 12 (2020): 911-925.

14. Hegarty K., et al. "Health practitioners' readiness to address domestic violence and abuse: A qualitative meta-synthesis". PLoS ONE 15.6 (2010): e0234067.

Volume 5 Issue 11 November 2021

(C) All rights are reserved by Naila Nasr Malik, et al. 